\title{
Emergency Surgery in a Critically Ill Patient with Major Drug-Induced Bleeding and Severe Ischaemic Heart Failure
}

\author{
Dana Tomescu1,2, Mihai Popescu1,2* \\ 1 "Carol Davila" University of Medicine and Pharmacy, Department of Anaesthesia and Critical Care, Bucharest, \\ Romania \\ ${ }^{2}$ Fundeni Clinical Institute, Department of Anaesthesia and Critical Care III, Bucharest, Romania
}

\begin{abstract}
Introduction: Anticoagulant overdose frequently occurs in elderly populations especially in remote areas where medical services are scarce. When emergency surgery is required, such patients offer major anaesthetic challenges.

Case presentation: We describe the case of an elderly patient admitted to a surgical ward with acute abdominal pain, on dual anti-platelet therapy and acenocoumarol for a recent acute myocardial infarction treated percutaneously with two drug-eluting stents. Laboratory tests showed severe anticoagulant overdose with uncoagulable INR. The decision was made to use of both light transmission aggregometry [LTA] for platelet function testing and thromboelastography to aid in the management of perioperative haemostasis in order to prevent both severe bleeding and stent thrombosis. Surgery revealed haemoperitoneum, volvulus of the ileum and a venous mesenteric infarction. Intraoperative blood loss was minimal and no blood products were administered. Postoperative course was uneventful without either thrombotic or haemorrhagic complications and the patient was discharged from the Postanaesthesia Care Unit on postoperative day two.
\end{abstract}

Conclusion: The use of aggregometry and thrombography helped in both evaluation and management of haemostasis of a high-risk patient by goal-directed administration of pro-and anti- coagulants.

Keywords: acenocumarol overdose, dual anti-platelet therapy, aggregometry, thromboelastography, emergency surgery

Received: 27 December 2016 / Accepted: 23 January 2017

\section{INTRODUCTION}

Due to an aging population, patients presenting for both elective and emergency surgery have more complex co-morbidities. Of these, heart disease represents the leading cause of death worldwide [1]. Nowadays, increasingly more elderly patients are treated with at least one antiplatelet drug concomitant with anticoagulants due to the high incidence of atrial fibrillation [2] and severe heart failure. These patients often miss their regular follow-up visits and so are at greater risk from oral anticoagulants misadministration and overdose compared to those that have regular follow-ups either with their general practitioner or cardiologist. It is not uncommon for such patients to present at a hospital with cerebral or gastrointestinal bleeding [3]. Reports on how to manage such patients have been extensively published recently $[4,5]$. When facing such a scenario, the anaesthesiologist must consider an individualized approach to preserve haemostatic balance without inducing pathological thrombosis or bleeding.

\section{CASE PRESENTATION}

The case of a 72-year-old male, weight $66 \mathrm{~kg}$, height $185 \mathrm{~cm}$, who presented to the emergency department with abdominal pain and constipation, is described. He complained of sharp pain, like "being stabbed", and that the symptoms had started four hours earlier. Physical examination showed a distended abdomen, tender, painful on palpation, especially in the mesogastric and hypogastric region, with a positive Blumberg sign, ir- 
regular tachycardic rhythm (115 beats/min), without increased blood pressure $(138 / 65 \mathrm{mmHg})$ and tachypnea (30 breaths/min). The patient was mildly agitated, with a shallow breathing pattern, pale, sweating and with cold extremities. His medical history showed he had permanent atrial fibrillation and had suffered an acute myocardial infarction three months previously. This was treated percutaneously with two drug-eluting stents on the anterior descending coronary artery. After revascularization, the left anterior descending coronary artery had a TIMI II flow. The right coronary artery had an $80 \%$ occlusion and was left untreated. Since then the patient was treated with acenocumarol and dual antiplatelet therapy (aspirin $100 \mathrm{mg} /$ day and clopidogrel $75 \mathrm{mg} /$ day).

An emergency abdominal computer tomography was performed for the preoperative differential diagnosis of acute abdominal pain and revealed thickening of the intestinal mucosa, volvulus of the small intestine and haemoperitoneum. Laboratory tests showed good liver and kidney function, leukocytosis, normal platelet count but non-coagulable blood tests (Table 1). Also a drop in haematocrit from $45 \%$, to $30 \%$ and in haemoglobin levels from $14.5 \mathrm{~g} / \mathrm{dL}$ to $9.8 \mathrm{~g} / \mathrm{dL}$ were noted compared with data taken at the time of the percutaneous coronary intervention. The patient confirmed that he was taking a tablet of acenocumarol daily without checking his INR and that he took both aspirin and clopidogrel on the day of hospital admission. A thromboelastogram (TEG 5000', Haemoscope, Niles, IL) was performed. (Figure 1A). The results were interpreted as either severe clotting impairment due to anticoagulant overdose or hyperfibrinolysis. Due to the high risk of antifibrinolytic therapy in a patient with a recent myocardial infarction and severe coronary artery disease it was decided to administer thirty units/kg of prothrombin complex concentrate (Pronativ ${ }^{\circledast}$, Octapharma, Manchester, UK). The TEG and standard coagulation
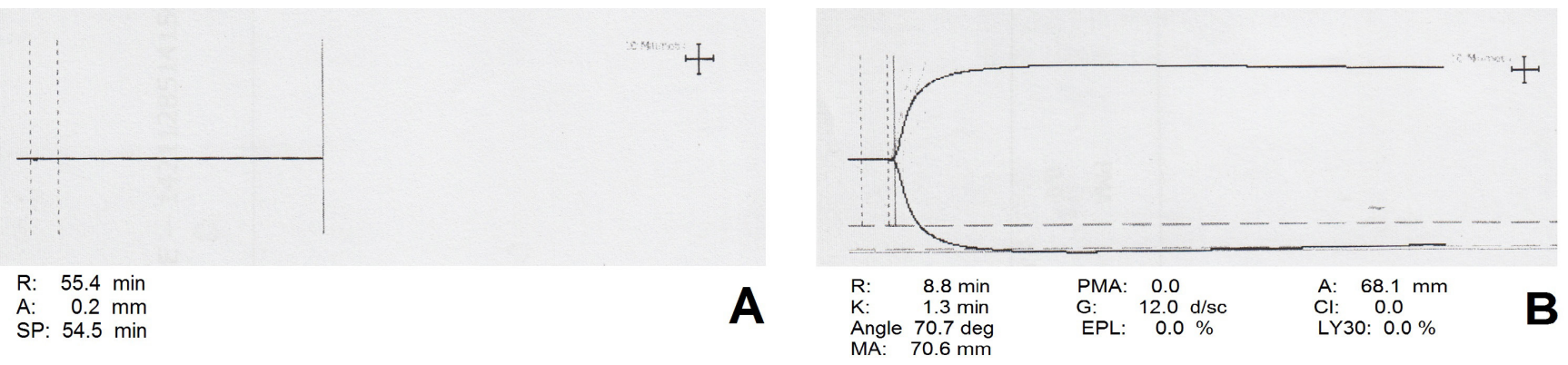

Fig. 1. Thromboelastogram at presentation (A) and after administration of $2000 \mathrm{U}$ prothrombin complex (B).A: severe clotting impairment due to anticoagulation overdose (flat line); B: near normal clotting activity (partial reversal of anticoagulation)

Table 1. Paraclinical results noted at presentation, after administration of prothrombin complex and 4 hours after surgery

\begin{tabular}{|c|c|c|c|}
\hline & At presentation & After PCC & After surgery (4 hours) \\
\hline $\operatorname{ALT}(U / L)$ & 21 & NA & 21 \\
\hline AST (U/L) & 27 & NA & 27 \\
\hline Total bilirubin (mg/dL) & 0.5 & NA & 0.5 \\
\hline Creatinine (mg/dL) & 0.91 & NA & 43 \\
\hline Blood urea nitrogen $(\mathrm{mg} / \mathrm{dL})$ & 40 & NA & 1.01 \\
\hline Troponin I (ng/mL) & 0.002 & NA & NA \\
\hline NTproBNP (pg/mL) & 2094 & NA & NA \\
\hline WBC $(* 103 / \mu \mathrm{L})$ & 12.56 & 15.84 & 16.78 \\
\hline Haemoglobin (g/dL) & 9.8 & 8.7 & 8.2 \\
\hline Haematocrit (\%) & 30.7 & 27.1 & 26.0 \\
\hline Platelet count $(* 103 / \mu \mathrm{L})$ & 317 & 308 & 261 \\
\hline aPTT (sec) & NC & 56 & 41.4 \\
\hline PT (sec) & NC & 418 & 24.1 \\
\hline INR & NC & 2.54 & 2.21 \\
\hline Fibrinogen (mg/dL) & 486 & 413 & 378 \\
\hline
\end{tabular}


tests were repeated (Figure 1B; Table 1) and showed near normal clotting activity with no signs of hyperfibrinolysis. Because of the risk of in-stent thrombosis and thromboembolic events the decision was taken to stop further administer of any pro-coagulant medication. Since the patient took both antiplatelet drugs that day an aggregometry (VerifyNow', Accumetrics, San Diego, CA, USA) was performed. The results showed a $20 \%$ inhibition for clopidogrel compatible with the profile of a partial responder.

Echocardiography showed interventricular septum, anterior and lateral wall akinesis with an ejection fraction of $30 \%$, which was unchanged compared to previous echocardiography that had been performed immediately after coronary re-vascularisation and a distended left atrium and grade III mitral regurgitation. Due to the severity of ischemic heart disease and the need for emergency surgery, the patient was classified as ASA IV E (American Society of Anaesthesiology Physical Status Classification). The patient was transferred to the surgical ward and emergency surgery was performed immediately.

A rapid sequence induction was performed. Intraoperative monitoring consisted of standard 2-lead electrocardiogram, pulse oximetry, temperature, bispectral index, train of four and invasive hemodynamic monitoring of central venous pressure, blood pressure and cardiac output by transpulmonary thermodilution (arterial catheter on left femoural artery)- PiCCO Plus System $^{\oplus}$, Pulsion Medical Systems, Feldkirchen, Germany. Maintenance of anesthesia was achieved with Sevoflurane titered to a minimum alveolar concentration between 1 and 1.2. The lungs were mechanically ventilated in pressure control to target a tidal volume of $6 \mathrm{~mL} / \mathrm{kg}$. An end-expiratory positive pressure of 5
$\mathrm{mmHg}$ was applied. The fresh gas flow consisted of $50 \%$ oxygen in air at a rate of two $\mathrm{L} / \mathrm{min}$. Bolus doses of fentanyl were administered during surgery at the anaesthesiologist's discretion and atracurium was used for neuro-muscular blockade. A cell salvage system and a rapid infusion system were prepared in case of massive blood loss.

Opening of the abdomen confirmed the diagnosis of haemoperitoneum with the presence of approximately $1000 \mathrm{ml}$ of fresh blood, and volvulus of the ileum. A venous mesenteric infarction associated with intestinal intramural hematomas as well as haematomas at the base of the mesentery were present. (Figure 2)

During surgery normovolemia was maintained using $2500 \mathrm{ml}$ of Ringer lactate and $1000 \mathrm{ml}$ of Gelofusine ${ }^{\oplus}$, in accordance with local protocol and PiCCO Plus data. There was no need for either vasopressor or inotropic support. No blood products were transfused during surgery. An enterectomy with terminal ileostomy was performed. On completion of surgery the trachea was safely extubated and the patient was transferred awake to the Postanaesthesia Care Unit. Postoperative anticoagulation was started six hours after surgery with unfractionated heparin to maintain an activated partial thromboplastin time between 50 and $70 \mathrm{sec}$ and converted 24 hours later to $6000 \mathrm{IU}$ of enoxaparin twice daily. Aspirin was re-started on postoperative Day One and Clopidogrel on postoperative Day Two. Routine echocardiography was performed on postoperative Day One and showed no changes compared with post re-vascularisation and preoperative assessments. Because the patient was only partially antiaggregated on clopidogrel a cardiologist was consulted who recommended maintenance of the previous dual-antiplatelet therapy and re-evaluation of therapy
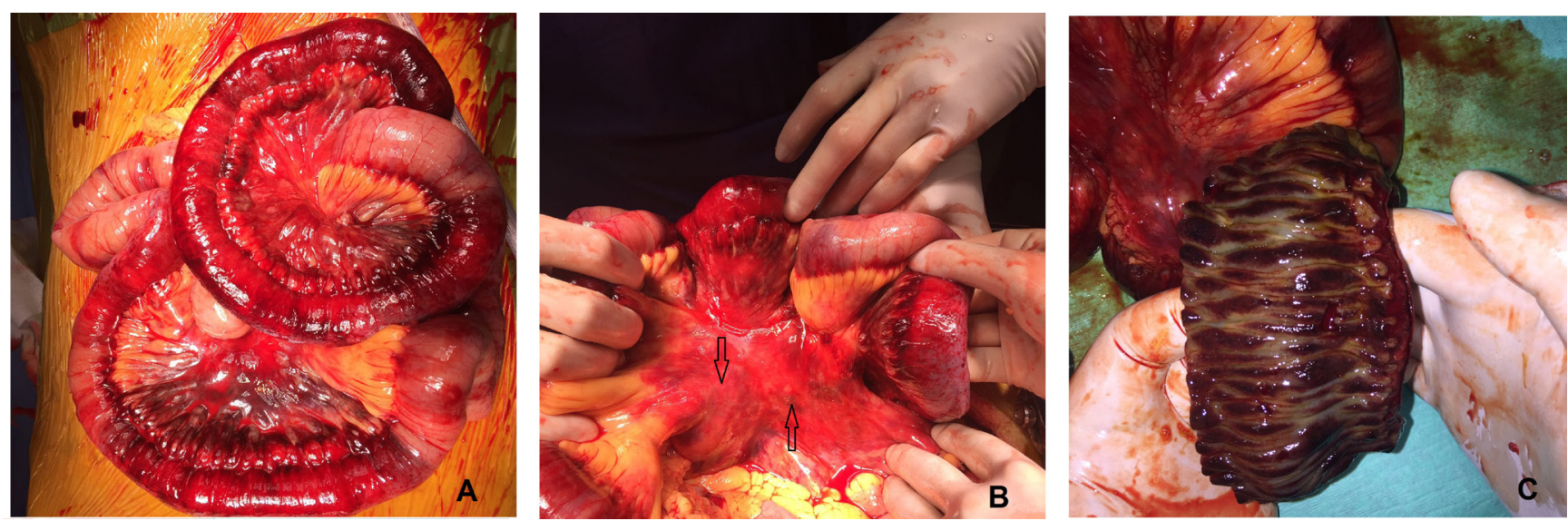

Fig. 2. Intraoperative findings. Venous infarction of the small intestine (A), hematomas at the base of the mesentery (B, arrows) and intramural and submucosal hematomas (C) 
one week after surgery. Postoperative course was uneventful and the patient was transferred to the surgical ward forty-eight hours after surgery.

\section{DISCUSSION}

The above case represents one of the most difficult clinical scenarios that an anaesthesiologist may encounter when managing haemostasis. Two major aspects must be considered. First, the risk of intraoperative massive blood loss in a patient that is actively bleeding and, second, the risk of perioperative myocardial infarction, stroke and in-stent thrombosis in a patient with a recent myocardial infarction and severe cardiovascular disease. On the other hand, the patient was intraoperatively diagnosed with mesenteric infarction that also required adequate anticoagulation. Hence, the particularity of this case relies on the association between venous mesenteric infarction, in a patient with coagulation deficit and bleeding status, and intestinal and mesenteric haematomas that needed emergency surgical intervention and intensive care management, to prevent both thrombotic and haemorrhagic complications.

Standard coagulation tests can be used for both dose adjustment and treatment of vitamin $\mathrm{K}$ antagonist overdosing, but such tests are not precise enough for patients taking multiple anticoagulants and antiplatelet drugs [6].High- risk patients, like the one presented, require fine tuning in order to optimize the haemostatic balance and prevent both bleeding and pathological thrombosis.

Viscoelastic coagulation testing represents an alternative to standard coagulation tests and offers a better and faster understanding of the haemostatic status. [7]. Moreover, thromboelastography has been used for the assessment of platelet reactivity in patients taking clopidogrel [8]. We used a combined approach. First, vitamin $\mathrm{K}$ antagonist overdose was diagnosed by standard coagulation tests. Both thromboelastography and point-of-care platelet function testing (VerifyNow ${ }^{\oplus}$ ) were used synchronously in order to assess the magnitude of oral anticoagulant overdose and antiaggregant activity. The $20 \%$ platelet inhibition by clopidogrel demonstrated that the major coagulation abnormality was due to acenocumarol overdose.

Fresh frozen plasma has been used for decades for oral anticoagulants overdose. This could not be applied in our case due to the high volume of fresh frozen plasma required. The administration of large volumes of plasma could have led to fluid overload and acute heart failure. Instead a prothrombin complex concentrate in accordance to recently published work was used [9]. The effects were assessed using thromboelastography. We aimed at achieving a partial reversal of anticoagulation (borderline normal) in order to prevent thrombotic complications. This approach was proven to be appropriate, both massive bleeding and thrombosis were avoided.

Recent guidelines suggest the use of new oral anticoagulants for patients at high risk of thrombosis and thromboembolism [10].Monitoring of such anticoagulants is not necessary and overdose can be effectively treated by using reversal agents. Current research offers promising results and probably, in the near future, anaesthesiologists will encounter fewer patients, if any, using vitamin $\mathrm{K}$ antagonists.

Patients with a coronary flow, only partially achieved after percutaneous coronary intervention, are at a high risk of major adverse cardiac events in the perioperative period [11]. In the case presented, the patient had low ejection heart failure, cardiac arrhythmia and TIMI II flow after re-vascularisation of a recent myocardial infarction. This, in conjunction with a severe bleeding (1000 $\mathrm{ml}$ haemoperitoneum), posed the possibility of a high risk of perioperative mortality.

In conclusion, emergency surgery in patients taking oral anticoagulants and dual antiplatelet therapy remains one of the greatest challenges an anaesthesiologist can encounter. Management of such patients requires precise diagnostics and fine tuning of drug therapy, including the use of point-of-care tests and prothrombin complex concentrates as treatment options. Perioperative intensive care should be aimed at both preventing further blood loss and pathological thrombosis.

\section{- Author Disclosure Statement}

The authors report no conflicts of interest.

\section{DEFERENCES}

1. Go AS, Mozaffarian D, Roger VL, Benjamin EJ, Berry JD, Borden WB, et al. Heart disease and stroke statistics--2013 update: a report from the American Heart Association. Circulation 2013;127:e6-e245.

2. Wilke T, Groth A, Mueller S, Pfannkuche M, Verheyen F, Linder $\mathrm{R}$, et al. Incidence and prevalence of atrial fibrillation: 
38 - The Journal of Critical Care Medicine 2017;3(1)

an analysis based on 8.3 million patients. Europace. 2013;15:486-93.

3. Liew A, Eikelboom JW, O'Donnell M, Hart RG. Assessment of Anticoagulation Intensity and Management of Bleeding With Old and New Oral Anticoagulants. Can J Cardiol. 2013;29:S3444.

4. Pernod G, Albaladejo P, Godier A, Samama CM, Susen S, Gruel $Y$, et al. Management of major bleeding complications and emergency surgery in patients on long-term treatment with direct oral anticoagulants, thrombin or factor-Xa inhibitors: proposals of the working group on perioperative haemostasis (GIHP). Arch Cardiovasc Dis. 2013;106:382-93.

5. Kozek-Langenecker SA, Afshari A, Albaladejo P, Santullano CA, De Robertis E, Filipescu DC, et al. Management of severe perioperative bleeding: guidelines from the European Society of Anaesthesiology. Eur J Anaesthesiol. 2013;30:270-382.

6. Favaloro EJ, Lippi G, Koutts J. Laboratory testing of anticoagulants: the present and the future. Pathology. 2011;43:682-92.

7. Blasi A, Muñoz G, de Soto I, Mellado R, Taura P, Rios J, et al. Reliability of thromboelastometry for detecting the safe coagulation threshold in patients taking acenocoumarol
Available online at: www.jccm.ro

after elective heart valve replacement. Thromb Res. 2015;136:669-72.

8. Yao Y, Zhang JH, Tang XF, He C, Ma YL, Xu JJ, et al. Head to Head Comparison of Two Point-of-care Platelet Function Tests Used for Assessment of On-clopidogrel Platelet Reactivity in Chinese Acute Myocardial Infarction Patients Undergoing Percutaneous Coronary Intervention. Chin Med J 2016;129:2269-74.

9. Sarode R, Milling TJ Jr, Refaai MA, Mangione A, Schneider A, Durn BL, et al. Efficacy and safety of a 4-factor prothrombin complex concentrate in patients on vitamin $\mathrm{K}$ antagonists presenting with major bleeding: a randomized, plasmacontrolled, phase IIIb study. Circulation. 2013;128:1234-43.

10. Kirchhof P, Benussi S, Kotecha D, Ahlsson A, Atar D, Casadei B, et al. 2016 ESC Guidelines for the management of atrial fibrillation developed in collaboration with EACTS. Europace. 2016;18:1609-1678.

11. Hawn MT, Graham LA, Richman JS, Itani KM, Henderson WG, Maddox TM. Risk of major adverse cardiac events following noncardiac surgery in patients with coronary stents. JAMA. 2013;310:1462-72. 